\title{
Niche and fitness differences relate the maintenance of diversity to ecosystem function
}

\author{
Ian T. Carroll, ${ }^{1,3}$ Bradley J. Cardinale, ${ }^{1,2}$ and Roger M. Nisbet ${ }^{1}$ \\ ${ }^{1}$ Department of Ecology, Evolution and Marine Biology, University of California, Santa Barbara, California 93106 USA \\ ${ }^{2}$ School of Natural Resources and Environment, University of Michigan, Ann Arbor, Michigan 48109 USA
}

\begin{abstract}
The frequently observed positive correlation between species diversity and community biomass is thought to depend on both the degree of resource partitioning and on competitive dominance between consumers, two properties that are also central to theories of species coexistence. To make an explicit link between theory on the causes and consequences of biodiversity, we define in a precise way two kinds of differences among species: niche differences, which promote coexistence, and relative fitness differences, which promote competitive exclusion. In a classic model of exploitative competition, promoting coexistence by increasing niche differences typically, although not universally, increases the "relative yield total," a measure of diversity's effect on the biomass of competitors. In addition, however, we show that promoting coexistence by decreasing relative fitness differences also increases the relative yield total. Thus, two fundamentally different mechanisms of species coexistence both strengthen the influence of diversity on biomass yield. The model and our analysis also yield insight on the interpretation of experimental diversity manipulations. Specifically, the frequently reported "complementarity effect" appears to give a largely skewed estimate of resource partitioning. Likewise, the "selection effect" does not seem to isolate biomass changes attributable to species composition rather than species richness, as is commonly presumed. We conclude that past inferences about the cause of observed diversity-function relationships may be unreliable, and that new empirical estimates of niche and relative fitness differences are necessary to uncover the ecological mechanisms responsible for diversity-function relationships.
\end{abstract}

Key words: biodiversity; coexistence; ecosystem function; MacArthur's consumer-resource model; niche and fitness differences; stabilizing and equalizing mechanisms.

\section{INTRODUCTION}

On a global scale, the contemporary decline in biodiversity is an uncontrolled experiment on biological influences over the cycles of energy and matter in whole ecosystems (Chapin et al. 1997, Sala 2000). On much smaller spatial and temporal scales, controlled experiments have shown that biodiversity could have a significant influence over a variety of functions performed by natural ecosystems (Tilman 1999, Loreau et al. 2001, Thompson and Starzomski 2007). Independent meta-analyses of research on biomass yield, an ecosystem function measured most commonly among primary producers but also among consumers and detritivores, confirm that reducing the number of trophically similar species typically reduces the efficiency of resource capture and utilization, which leads, in turn, to reductions in biomass within that trophic level (Balvanera et al. 2006, Cardinale et al. 2006, 2007, Stachowicz et al. 2007).

Manuscript received 10 February 2010; revised 29 October 2010; accepted 4 November 2010. Corresponding Editor: S. J. Schreiber.

${ }_{3}^{3}$ E-mail: carroll@lifesci.ucsb.edu
Theories explaining positive correlations between species richness and biomass are frequently rooted in the idea of the ecological niche, a notoriously difficult concept to define and measure (Leibold 1995). Over a decade ago, Tilman et al. (1997) popularized the notion that niche partitioning among competitors should yield positive effects of diversity on biomass. Quantitative study of niche partitioning has since relied on either species' traits or their relatedness. Experiments that manipulate functional trait diversity along with species richness offer a coarse approach to capturing the role of ecological niches in diversity-biomass relationships (Hooper and Vitousek 1997, Díaz and Cabido 2001). More recently, phylogenetic distance has outperformed functional trait diversity as a predictor for the correlation between richness and biomass in plant communities, although it is unknown whether phylogenetic distance predicts trait differentiation (Cadotte et al. 2008).

Correlations between species richness and community biomass do not, however, require niche differentiation. Originally thought to be a statistical artifact (Aarssen 1997, Huston 1997), correlations that are now recognized as "selection effects" (Loreau and Hector 2001) or "dominance effects" (Fox 2005) of diversity result when 
species that have either above- or below-average biomass are also competitively dominant. With any particular species more likely to be chosen as a greater number of species are randomly selected from a common pool, high-diversity treatments tend toward the biomass of any species able to exclude its competitors. Coupled with variability in monoculture biomass, competition can lead to either positive or negative correlations between diversity and community biomass. To discern whether niche differences or competitive dominance cause the observed correlations between diversity and biomass, many researchers rely on "additive partitions" of biomass data, but even the authors of these methods are cautious about using them to infer the strength of particular biological mechanisms (Fox 2005, Hector et al. 2009).

Diversity-function researchers are not the first to entertain the idea of distinguishing competitive dominance from niche partitioning. Efforts to untangle the two have a much longer history in coexistence theory, as knowledge of each is required to understand the maintenance of species diversity (Chesson 2000). A case in point is the nearly identical figure published by MacArthur and Levins (1967: Fig. 2), May (1974: Fig. 6), and Chesson (1990: Fig. 2), which shows that the region in one model's parameter space compatible with competitive coexistence depends on both niche overlap and the degree of dominance. The metrics used in each figure vary, but the argument is consistent: a niche difference must be large enough to overcome the relative difference in fitness for species to coexist. In Chesson's (2000) terms, stabilizing mechanisms (which increase the niche difference) and equalizing mechanisms (which reduce the relative fitness difference) are distinct mechanisms that both promote coexistence.

Because the niche difference (ND) and relative fitness difference (RFD) between competitors are key ingredients for understanding both the coexistence of competitors as well as the relationship between biodiversity and community biomass, they could potentially bridge two bodies of theory and experiments in community ecology. Ecosystem-level consequences of extinction may be predictable in communities whose coexistence mechanisms are known (Mouquet et al. 2002). Alternatively, if the results from diversity manipulations also provide information on the magnitude of the ND and RFD, they could help to identify the relative importance of niche and neutral processes in maintaining diversity (Adler et al. 2007). Despite the ubiquity of the ND and RFD concepts, a standard method for taking their measure remains allusive. We find Chesson's (2000) approach, which invokes their effect on mutual invasion rates, compelling. To our knowledge, however, no general method to unambiguously calculate a ND and RFD for multispecies competition has been defined in the literature.

Here, we contribute to theory relating species coexistence to diversity's effect on ecosystem function by reporting how consumer biomass varies as a result of both the ND and RFD in the consumer-resource model described by MacArthur (1970). So that experimental and theoretical work on this and other models can be exactly comparable, we suggest a model-independent definition of niche and relative fitness differences. Thus we begin by reinterpreting Chesson's (2000) framework to propose a precise measure of the ND and RFD that applies to a broad range of competitive systems. In an analytically tractable case of MacArthur's model, our procedure for calculating the ND and RFD yields the same values reported by Chesson $(1990,2000)$. We then relate these measures to the "relative yield total," an index commonly used to quantify the effect of diversity on biomass, among MacArthur's consumers. Finally, we discuss Loreau and Hector's (2001) additive partition in light of the relationship shown to exist between the relative yield total and the niche and fitness differences among competitors.

\section{Niche And Fitness Differences}

MacArthur's consumer-resource model has had a large influence on the theory of exploitative competition, undoubtedly due to its mathematically tractable form. Despite many early and valid criticisms (e.g., Abrams 1975, Schoener 1976), the model is an historically significant one that we use to illustrate our modelindependent definitions of niche and relative fitness differences. Using the notation of Chesson (1990), MacArthur's model defines the dynamics of $q$ biotic resources $\left(R_{i}\right)$ and $n$ consumers $\left(X_{i}\right)$ by the following equations:

$$
\begin{array}{ll}
\frac{d R_{i}}{d t}=R_{i} r_{i}\left(1-\frac{R_{i}}{K_{i}}\right)-\sum_{j=1}^{n} c_{j i} R_{i} X_{j} & \text { for } i=1 \ldots q \\
\frac{d X_{i}}{d t}=X_{i} b_{i}\left(\sum_{j=1}^{q} c_{i j} w_{j} R_{j}-m_{i}\right) & \text { for } i=1 \ldots n .
\end{array}
$$

The equations convey that the $i$ th resource species grows logistically at an initial rate, $r_{i}$, to a carrying capacity, $K_{i}$, in the absence of consumers. When present, the $i$ th consumer exhibits a linear functional response, reducing resources at a constant per capita rate, $c_{i j}$ (of matrix c), while assimilating biomass according to the factor $w_{j}$. Assimilated biomass is lost at specific rate $m_{i}$, due to mortality, metabolism, or any other loss process, and what remains is converted, by the factor $b_{i}$, to new consumer biomass.

The niche difference (ND) for consumers in MacArthur's model corresponds to differences in resource utilization, or more precisely the differences among the rows of matrix c, which define each consumer's per capita consumption rate on every resource. However, the exact measure of the degree of this difference has historically varied among researchers. MacArthur and Levins (1967) and May (1974) assumed that consumers and resources are spaced evenly along one or more niche 
axes, allowing the niche difference to be a parameter for the distance between peaks of each consumer's Gaussian utilization function. Chesson (1990) did not need such strong assumptions, arguing instead that the niche difference between two consumers could be described statistically. He showed that if we define $a_{i j}$, the symmetrical competition coefficient involving consumers of type $i$ and $j$, as $a_{i j} \equiv \sum_{h=1}^{q} c_{i h} c_{j h}\left(w_{h} K_{h} / r_{h}\right)$, then a weighted, zero-intercept regression of the $i$ th row against the $j$ th row of $\mathbf{c}$ has a coefficient of determination, denoted by $\rho^{2}$ equivalent to $a_{i j} a_{j i}\left(a_{i i} a_{j j}\right)^{-1}$. A value of $\rho^{2}$ near 1.0 indicates strong similarity between two consumers in their consumption of the various resources, creating a minimal niche difference. Alternatively, a value of $\rho^{2}$ near 0 indicates weak overlap in the consumers' diet and thus a strong niche difference.

The strength of the ND alone does not determine whether consumers governed by Eq. 1 will coexist: whether or not superior competitors exclude species with sufficiently similar niches also depends on a relative fitness difference (RFD). MacArthur and Levins (1967), May (1974), and Chesson (1990) each implicitly defined a RFD and found regions of the ND and RFD plane that permit coexistence among competitors. In our notation, the measure of relative fitness given by Chesson (1990) is, $k_{i} / \sqrt{a_{i i}}$, where $k_{i} \equiv \sum_{h=1}^{q} c_{i h} w_{h} K_{h}-$ $m_{i}$. By this definition, a consumer's relative fitness includes its response to factors limiting growth as well as its effect on those limiting factors, which are two essential elements of population regulation (Leibold 1995, Meszena et al. 2006). The value of $k_{i}$ is the consumer's ability to utilize resources for growth in the absence of any competition. The value of $\sqrt{a_{i i}}$ is the magnitude of the consumer's vector of weighted resource capture rates. The ratio between the two, a measure of the maximum rate of net biomass assimilation per unit of resource depletion, is a defensible measure of consumer efficiency. Chesson's definition of relative fitness may also be justified by noting that two consumers will stably coexist with two resources if and only if $\rho<\left(k_{2} / \sqrt{a_{22}}\right)\left(\sqrt{a_{11}} / k_{1}\right)<\rho^{-1}$ (a classic result, but see Haygood [2002] for a recent proof with extensions).

A quite general procedure we now set out for the calculation of the ND and RFD, although not limited to MacArthur's model, arrives at these same measures for a special case of Eq. 1. The procedure defines the ND and RFD not by species' traits, but by an intuitive connection to the population dynamics that result from competition. The type of competition is not restricted and includes competition for biotic or abiotic resources, space, or escape from natural enemies, as well as direct interference, potentially admitting applications of the procedure beyond the realm of the exploitative niche. Following Chesson (2000), the procedure begins with consideration of mutual invasion rates, or change in the population size of each competitor as it invades a set of established residents after having been eliminated. We caution that our treatment is not completely general for reasons discussed briefly following the definitions and at greater length in Appendix A.

Assume that the population dynamics for a set of $n$ competitors are solutions to $\dot{N}_{i}=N_{i} g_{i}(\mathbf{N})$ for $i=1 \ldots n$, where the per capita growth rate, $g_{i}$, is a scalar function of the vector of state variables, $\mathbf{N}$. The dimension of $\mathbf{N}$ may exceed $n$ when growth rates depend on variables other than the $n$ competitors. In Eq. 1, for example, $\mathbf{N}$ also includes resource levels, so specifying the full community requires more than $n$ equations. Assume also that the system approaches an equilibrium for each state variable in the absence of all $n$ competitors, denoted by the vector $\mathbf{O}$, and that any one competitor would thrive without competition (i.e., $g_{i}(\mathbf{O})>0$ for all $i)$. To obtain an invasion rate for species $i$, remove it from the system by setting $N_{i}=0$ and find an equilibrium, denoted by vector $\mathbf{I}_{-i}$, approached by the remaining state variables (i.e., in the absence of only one of the $n$ competitors). Near this equilibrium, $\dot{N}_{i} \approx$ $N_{i} g_{i}\left(\mathbf{I}_{-i}\right)$ by a first-order Taylor expansion, and the equilibrium is said to be invasible if $g_{i}\left(\mathbf{I}_{-i}\right)$ is positive. Mutual invasibility, or having positive values of $g_{i}\left(\mathbf{I}_{-i}\right)$ for all $i$, may lead either to permanent coexistence (Armstrong and McGehee 1980) or to an "endless cycle of extinctions and reinvasions" (Jansen and Sigmund 1998). In either case, comparing an invader's growth rate, $g_{i}\left(\mathbf{I}_{-}\right)$, to its growth rate without competitors, $g_{i}(\mathbf{O})$, reveals how much the invader suffers from interspecific competition.

We argue that the ND can be defined by an average effect of interspecific competition on invaders, and that the RFD can be equated to the variability in these effects. Niche differences, in a broad sense, are any property of an ecological community that relaxes competition between different species, and this increases the average invasion rate. Likewise, variability among invasion rates reflects the differential sensitivity to interspecific competition within a community. Competitive exclusion is just a sufficiently large difference in invasion rates. Thus, without specifying the form of interactions among species, we will measure the ND and RFD by the effect of the interactions on population dynamics: specifically, invasion rates.

The proportional reduction in an invader's growth rate due to interspecific competition will be called its sensitivity, $S_{i}$, defined thus:

$$
S_{i} \equiv \frac{g_{i}(\mathbf{O})-g_{i}\left(\mathbf{I}_{-i}\right)}{g_{i}(\mathbf{O})} .
$$

Here, the difference between $g_{i}(\mathbf{O})$ and $g_{i}\left(\mathbf{I}_{-i}\right)$ is standardized so that variation among the $S_{i}$ values does not just reflect differences in the characteristic timescale of each population's growth rate. The $S_{i}$, unlike the raw growth rates, are dimensionless. The lack of units facilitates comparison not only between competitors but also between species in different ecosystems or experimental communities. We limit the scope of 
analysis to true competitors, or those with $g_{i}\left(\mathbf{I}_{i}\right)$ less than $g_{i}(\mathbf{O})$, so $S_{i}$ has a minimum of 0 . Importantly, this assumption does not exclude interspecific facilitation, and our results hold as long as the combined effect of the resident community on the invader is to reduce its population growth rate.

Finally, we summarize the set of $S_{i}$ by their geometric mean and geometric standard deviation. Because a niche difference reduces competition, corresponding to a decrease in the $S_{i}$, we make the following explicit definitions:

$$
\mathrm{ND} \equiv 1-\prod_{i=1}^{n} S_{i}^{1 / n}
$$

and

$$
\mathrm{RFD} \equiv \exp \left[\left(\overline{(\ln S)^{2}}-\overline{\ln S^{2}}\right)^{1 / 2}\right]
$$

where the exp and $\ln$ functions are used to obtain the geometric standard deviation from the arithmetic means indicated by an overbar. The geometric mean responds in a useful way to values of $S_{i}$ approaching 0 , corresponding to species highly tolerant of competition. A small change near 0 has a larger effect on the geometric mean than an equally small change closer to 1 , so competitively dominant species carry greater weight than more sensitive species.

For a special case of MacArthur's consumer-resource equations, the procedure just described obtains the same expressions deduced by Chesson $(1990,2000)$ from the specific form of the model. An analytical expression for the invasion rates for two consumers of $q$ resources in terms of $a_{i j}$ and $k_{i}$ is only possible when none of the resources are driven to extinction by the resident consumers (Abrams et al. 2008). Assuming that the parameter values chosen permit positive resource equilibria, carrying out our procedure for two of MacArthur's consumers yields ND $=1-\rho$ and RFD $=\left(k_{2} / \sqrt{a_{22}}\right)\left(\sqrt{a_{11}} / k_{1}\right)$, where without loss of generality we let consumer 2 be the one with greater relative fitness. Our procedure remains applicable beyond this special case; we only lose the ability to write down a solution for the ND and RFD in closed form.

With the procedure defined, we can now describe its limitations. Along with proposing that coexistence mechanisms may be described as either equalizing (reducing the RFD) or stabilizing (increasing the ND), Chesson (1994) has fully defined a partition between mechanisms that work in systems that approach steady states and mechanisms that require populations to fluctuate. Our approach relies on a first-order approximation to the low-density growth rate, so one limitation is that only the fluctuation-independent mechanisms are measured. A second limitation emerges in systems with multiple attractors, which would create multiple values for each $S_{i}$ and render our measures nonunique. Third, there are forms of competition in which mutual invasibility does not guarantee permanent coexistence, such as intransitive competition (sensu Edwards and Schreiber 2010). For these cases, interpretation of the ND and RFD is considerably more challenging. Appendix A elaborates these points, none of which stands in the way of continuing our analysis of the simple model of MacArthur (Haygood 2002).

\section{Effect of Diversity on Consumer Biomass}

We now turn our attention toward showing how the differences between species that influence coexistence also control the effect of biodiversity on biomass yield among MacArthur's consumers. We employ the widely used "relative yield total" to quantify the influence of consumer diversity on consumer biomass at equilibrium (Vandermeer 1989, Hector 1998). The relative yield of an individual consumer is the ratio of its equilibrium biomass in a multispecies community, $X_{i}^{*}$, to the biomass it achieves when grown in monoculture, $M_{i}$. Summation over all species gives the relative yield total: $\mathrm{RYT} \equiv \sum_{i=1}^{n} X_{i}^{*} / M_{i}$. Individual relative yields are less than 1 in competitive communities, and the RYT of a polyculture approaches the total number of species as each one approaches its monoculture biomass.

Beginning with the same special case of MacArthur's equations, when neither consumer can independently drive resources to extinction, the RYT for coexisting species can be expressed exactly in terms of the ND and RFD (Appendix B):

$$
\mathrm{RYT}=\frac{2-(1-\mathrm{ND})\left(\mathrm{RFD}+\mathrm{RFD}^{-1}\right)}{\mathrm{ND}(2-\mathrm{ND})} .
$$

Fig. 1A shows how variation in the RYT relates to the strength of the ND and RFD, as given by Eq. 4. When the RFD overcomes the ND (blank region of Fig. 1A), one consumer is competitively excluded and the RYT would equal 1. As shown by Loreau (2004) for an equivalent Lotka-Volterra model, coexistence alone guarantees an RYT $>1$. However, we can extend this result by observing that both equalizing (reducing the RFD) and stabilizing (increasing the ND) mechanisms of diversity maintenance contribute jointly to the positive effect of diversity. Although there is no surprise in finding that increasing the ND both favors coexistence and increases the relative yield total, we did not anticipate that making competitors more similar by reducing their RFD would have a similar impact: favoring coexistence while also increasing the relative yield total.

To assess whether results from the analytically tractable case just described might also be typical, we used a Monte Carlo method to examine MacArthur's model under less restrictive parameter constraints (see Appendix $\mathrm{C}$ for a description of random parameter generation). Among communities with two consumers and two resources, now allowing resources to go extinct, the variation in RYT remains tightly controlled by both the ND and RFD (Fig. 1B). This suggests that the ND 


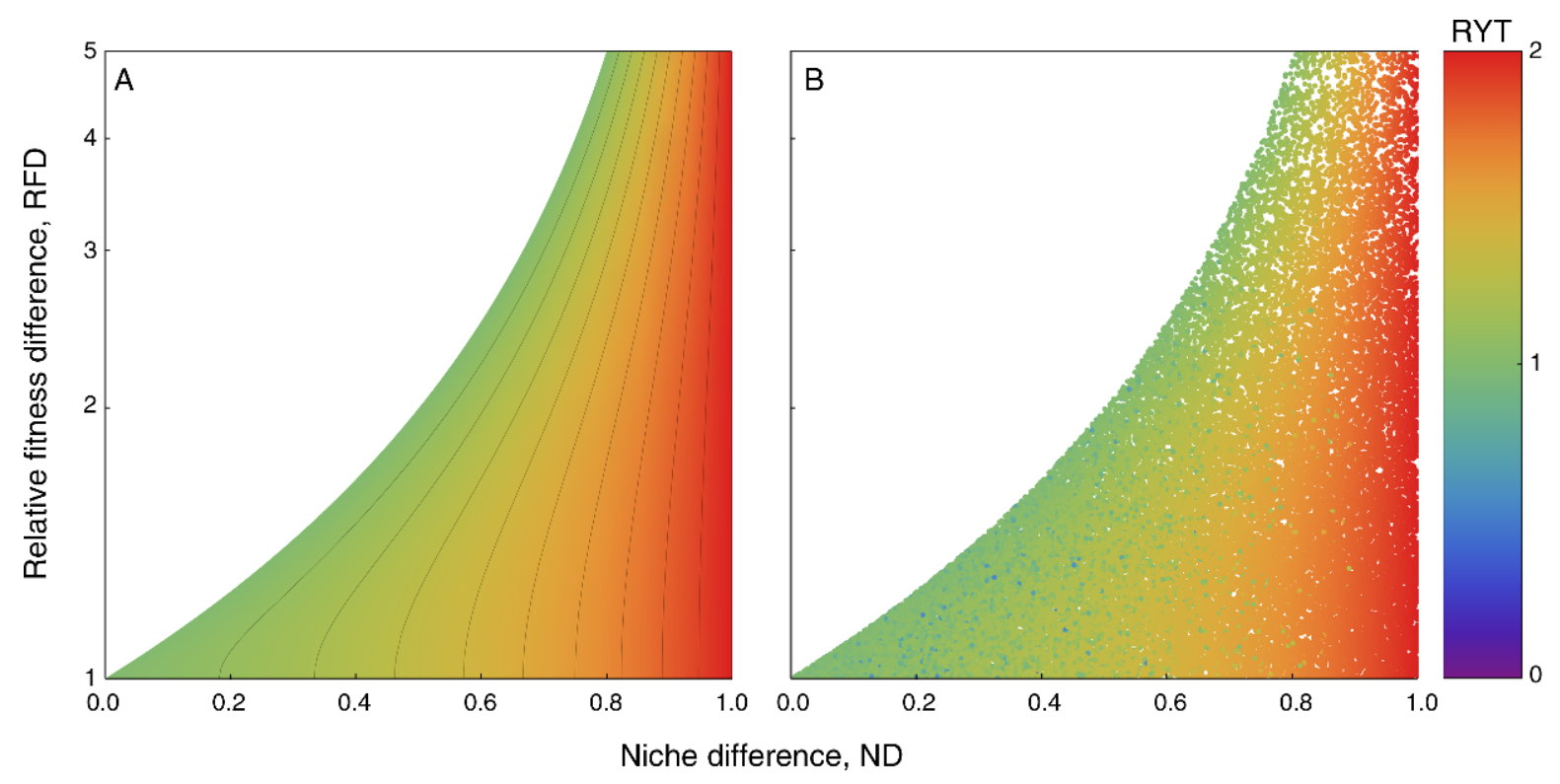

FIG. 1. The relative yield total (RYT, color key) for two consumers governed by Eqs. 1a and $1 \mathrm{~b}$ plotted as a function of their niche and relative fitness difference. RYT is a measure of diversity's effect on the biomass of competitors. (A) Analytical solution corresponding to Eq. 4, which is valid when resources do not go extinct. (B) Numerical results in which each colored dot corresponds to one of 40000 communities with parameters selected at random (see Appendix C).

and RFD interact to regulate the impact of consumer diversity on biomass yield over a broad range of parameter values. We observe, however, that in cases where the resident consumer overexploits a resource, driving it to extinction, the RYT tends to be lower than that given by Eq. 4. In fact, unlike the preceding special case without extinctions, it is possible for consumers to coexist with RYT $<1$, which is interpreted as a negative effect of diversity on biomass. Extinction among MacArthur's resources introduces a nonlinearity that can drastically reduce the RYT, a point we will return to in our discussion.

To assess whether our results are sensitive to the number of consumer species, we calculated RYTs for additional communities having three and four coexisting consumers. In all cases, increasing the ND and decreasing the RFD are both associated with stronger positive effects of diversity on biomass. As a crude indicator of this association, linear least-squares regression on $\mathrm{RYT} / n$ consistently returns a positive coefficient for the ND, a negative coefficient for the RFD, and large adjusted- $R^{2}$ values (Table 1 ). The relative magnitude of the coefficients confirms what is visually apparent in Fig. 1; the gradient in RYT is steeper in the ND direction. Although suggestive, this result does not reliably quantify how much greater in importance the ND becomes for regulating the RYT. Not only are the ND and RFD strongly correlated (a result of constraining their values to allow coexistence), but also we have to admit little knowledge of the realistic range of variation in either difference that could be expected in natural or experimental communities (Adler et al. 2007).
To summarize our results for the impact of the ND and RFD on consumer biomass, we conclude that two distinct mechanisms that maintain diversity also tend to increase the effect of diversity on the total relative yield of consumers. Based on prior studies, we might have suspected that competitive dominance would only increase the relative yield total through some form of "selection effect," whereby a species with high yield in monoculture tends to dominate more diverse communities. However, in MacArthur's model the impact of the RFD is more pervasive than expected. Fitness differences also interact with niche differences, diminishing the ability of a suite of consumers, in which some populations are more suppressed by competition, to effectively utilize all available resources or the entire niche space.

\section{Relevance to Diversity Experiments}

Our demonstration of a novel role for the RFD in a diversity-function relationship, along with our precise measure of the ND, yield some insight on the interpretation of results from biodiversity manipulations. One goal of diversity-function research is to predict how diversity per se affects ecosystem functions, irrespective of the composition of the community. Given the uncertainty over which species may vanish from an ecosystem, a useful first step is to distinguish the impact of random species loss on community biomass from the impact of a particular species' extinction. To this end, Loreau and Hector (2001) proposed that the results of diversity manipulations could be interpreted in terms of the average deviation in yield for each species from a 
TABLE 1. Results for a linear regression predicting RYT $/ n$ from ND and $\ln$ RFD for $n$ consumers of $n$ resources governed by Eq. 1, with parameters selected at random.

\begin{tabular}{|c|c|c|c|c|c|}
\hline \multirow[b]{2}{*}{$n$} & \multirow[b]{2}{*}{ Adjusted $R^{2}$} & \multirow[b]{2}{*}{ Predictor } & \multirow[b]{2}{*}{ Coefficient } & \multicolumn{2}{|c|}{$95 \% \mathrm{CI}$} \\
\hline & & & & Lower & Upper \\
\hline \multirow[t]{3}{*}{2} & 0.827 & intercept & 0.358 & 0.357 & 0.360 \\
\hline & & ND & 0.619 & 0.616 & 0.622 \\
\hline & & ln RFD & -0.107 & -0.108 & -0.105 \\
\hline \multirow[t]{3}{*}{3} & 0.872 & intercept & 0.235 & 0.234 & 0.237 \\
\hline & & ND & 0.741 & 0.738 & 0.744 \\
\hline & & $\ln \mathrm{RFD}$ & -0.110 & -0.112 & -0.107 \\
\hline \multirow[t]{3}{*}{4} & 0.894 & intercept & 0.162 & 0.161 & 0.163 \\
\hline & & ND & 0.818 & 0.814 & 0.822 \\
\hline & & ln RFD & -0.117 & -0.120 & -0.114 \\
\hline
\end{tabular}

Notes: The first predictor is 1; intercept refers to the regression coefficient associated with 1 . RYT is relative yield total; niche difference (ND) and relative fitness difference (RFD) between competitors are key ingredients for understanding coexistence and the relationship between biodiversity and community biomass. Sample size is 40000 , with $P<0.001$ for each diversity level. See Appendix $\mathrm{C}$ for details.

null expectation of no diversity effect, $\overline{\Delta Y}$. For each species in a polyculture, $\Delta Y_{i}$ is the product of its monoculture biomass, $M_{i}$, and its deviation from the null expectation for its relative yield, $\Delta \mathrm{RY}_{i}$. On taking expectations, the product of means $\bar{M} \overline{\Delta \mathrm{RY}}$ and the covariance $\operatorname{Cov}(M, \Delta \mathrm{RY})$ form an additive partition of $\overline{\Delta Y}$. Loreau and Hector (2001) called $n \bar{M} \overline{\Delta R Y}$ the "complementarity effect" and $n \operatorname{Cov}(M, \Delta \mathrm{RY})$ the "selection effect." It is now common practice to assume that the first is caused by niche partitioning or facilitation and that the second is driven by competitive dominance.

In contrast to the common interpretation, we find that both the complementarity and selection effects are an amalgam of the interactions measured by the ND and RFD. It is straightforward to perform the additive partition for the special case of MacArthur's model that allowed a closed-form solution for the RYT (Eq. 4). We assume, without affecting our conclusion, that the null expectation for the relative yield of each species has the value $1 / n$, which is normal in empirical research. The complementarity effect among two consumers expressed in terms of their sensitivity to competition and the ND is then

$$
\bar{M}\left(\frac{2}{2-\mathrm{ND}} \frac{1-\bar{S}}{1-\sqrt{S_{1} S_{2}}}-1\right)
$$

and the selection effect may be calculated as

$$
\frac{2 \operatorname{Cov}[M, S]}{\mathrm{ND}(2-\mathrm{ND})} \text {. }
$$

For this special case, expression 5 reveals that any variability in relative fitness between consumers, regardless of its correlation with monoculture biomass, reduces the complementarity effect. The arithmetic mean of the sensitivity to competition, $\bar{S}$, is always greater than or equal to its geometric mean, $\sqrt{S_{1} S_{2}}$, so for a given ND, expression 5 is maximized when the $S_{i}$ are equal, corresponding to no fitness difference. Additionally, expression 6 shows that the magnitude of the selection effect depends on the amount of niche partitioning, along with the covariance between the $S_{i}$ and monoculture biomass. In short, the additive partition does not isolate the effects of biodiversity on total biomass due to the ND from those of the RFD.

Although the ND and RFD are clearly not mathematically equivalent to the complementarity and selection effects, only by comparing their values in numerical simulations do we get a qualitative picture of how they diverge. The complementarity effect has units of biomass yield, but scaling it by the average monoculture yield, $\bar{M}$, leaves just (RYT -1 ) (Loreau and Hector 2001). Because the RYT has a maximum of $n$, further standardizing the complementarity effect by $(n-1)$ puts it on the same scale as the ND for any diversity level. Fig. 2 shows that the rescaled complementarity effect can give a first approximation to the ND, with $R^{2} \geq 0.75$ for all three diversity levels (Fig. 2). The association, however, is not $1: 1$, as the ND is consistently underestimated by the rescaled complementarity effect. Knowledge of the complementarity effect does not, therefore, allow even an approximation of the ND without some a priori knowledge of the regression coefficients.

We emphasize these facts because, despite criticism and arguments to the contrary (Petchey 2003, Fox 2005, Cardinale et al. 2007, Hector et al. 2009), a persistent notion in the literature on diversity-function research is that the complementarity effect directly quantifies the extent of niche partitioning and/or facilitation among species. We find this interpretation imprecise and argue that continued reliance on the complementarity effect does not advance the goal of resolving the biological mechanisms responsible for diversity-biomass relationships. In addition, we do not support the claim that selection effects measure the amount of biomass lost or gained due to dominant competitors. Anything less than perfect niche partitioning inflates the selection effect given by expression 6, so the covariance between 
competitively dominant species and their monoculture biomass cannot be related to the selection effect without knowledge of the ND.

None of our arguments imply that empirical measurements of biological mechanisms that influence diversity-function relationships, as summarized by the ND and RFD, are unobtainable. To the contrary, the procedure we proposed to measure the ND and RFD in a theoretical context is directly transferrable to an empirical one. Niche and relative fitness differences among species are calculated directly from certain population growth rates, which can be empirically measured when the necessary experimental manipulation is feasible. If an empiricist can measure an invading population's growth rate following (1) its introduction into an established community at steady-state biomass and (2) into a competitor-free habitat, then the subsequent calculations of the ND and RFD are straightforward. The proportional reduction in population growth rate of a rare species between treatments (1) and (2) allows a calculation of that species' sensitivity to competition (Eq. 2). Duplicating the experiment with different species as the invader provides samples from the distribution of sensitivity to competition within the community. The geometric mean and standard deviation of this distribution then defines the niche and relative fitness differences, respectively (Eq. 3).

We recognize that it is infeasible to measure invasion dynamics in many biological communities. For example, researchers must measure the growth rates of focal populations, rather than the growth of individuals (which is often not done in biodiversity studies, e.g., in grasslands). Additionally, resident species must be maintained near a multispecies equilibrium, so that the invading species can be introduced at low density into a preestablished community. However, we point out that such conditions can be met in laboratory systems (e.g., Warren et al. 2003), and related designs have already been proposed for quantifying the effects of niche and relative fitness differences on coexistence in field experiments (Adler et al. 2007). Empirical results from such studies demonstrate that both kinds of differences among species are important drivers of coexistence (Harpole and Suding 2007, Levine and Hille Ris Lambers 2009), and similar designs could empirically detect the impact of any ND and RFD on diversityfunction relationships. These experiments easily could be performed in tandem with manipulations of species richness, providing the data to show whether and how the two mechanisms of coexistence also control the influence of biodiversity on ecosystem function.

\section{Discussion}

We began by proposing a broadly applicable method for measuring the niche difference (ND) and relative fitness difference (RFD) between competitors, refining a framework for understanding mechanisms that maintain species diversity (Chesson 2000). We then asked how the

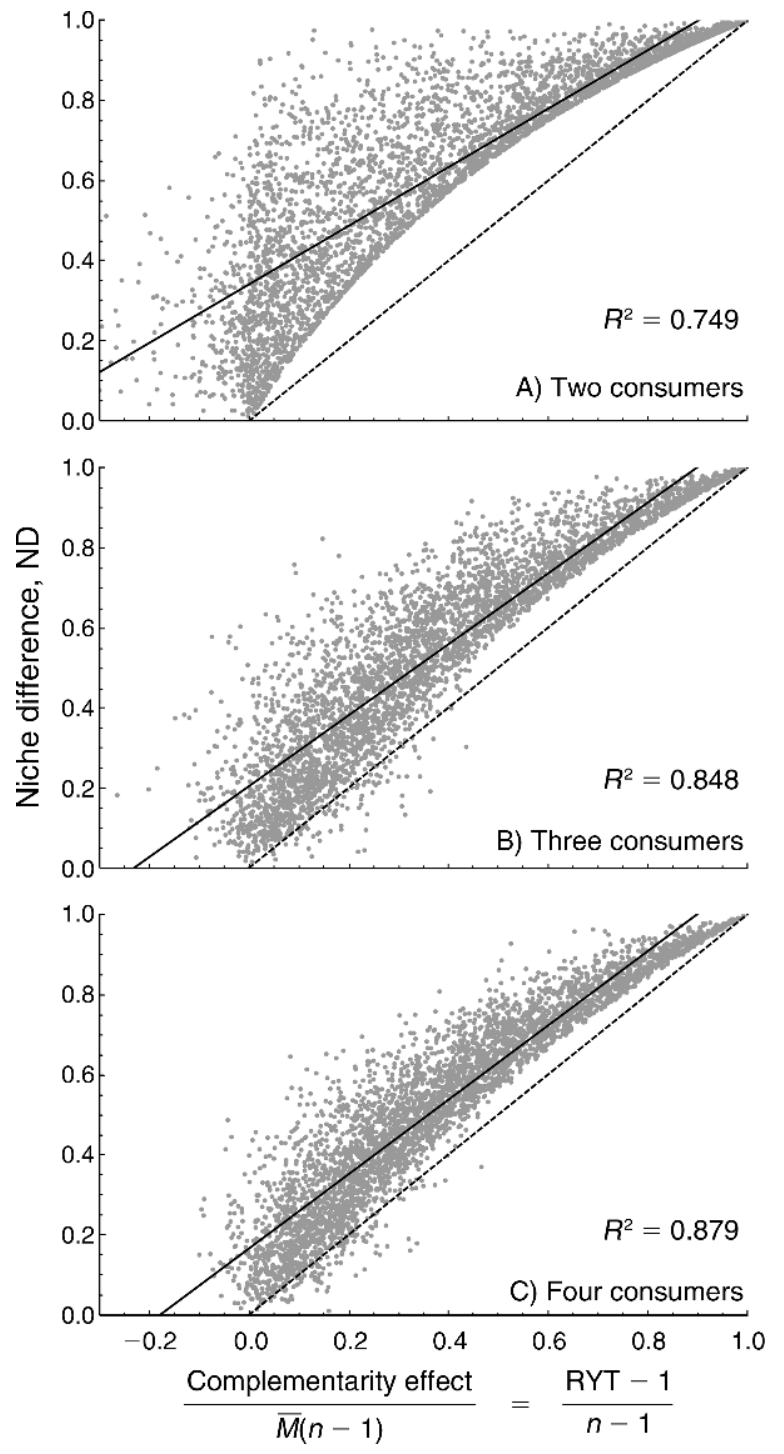

FIG. 2. The predicted niche difference (ND) from linear regression against $(\mathrm{RYT}-1) /(n-1)$, a rescaled complementarity effect (solid line), with the 1:1 (dotted) line shown for comparison. Gray dots are a representative sample of the 40000 simulated communities in the regression.

ND and RFD relate to the effect of species richness on an ecosystem function, specifically the relative yield total of biomass for a single trophic level. For a restricted case of MacArthur's consumer-resource model, we gave an exact relationship between two general mechanisms that promote coexistence and the effect of diversity on biomass yield. For more general cases, with up to four consumer species, we numerically demonstrated a similar relationship: both increasing the ND and decreasing the RFD typically increase this effect of diversity. We then compared the ND and RFD in MacArthur's model to additive partitions of biodiversity-function experiments, and argued that the latter are frequently misinterpreted. However, we also noted how 
experiments could better link coexistence mechanisms to ecosystem function.

Although niche partitioning has long been perceived as the sole mechanism guaranteed to increase the relative yield total as new species are introduced to experimental communities, we showed that equalizing the relative fitness differences between consumers can have the same effect. This suggests that competitive dominance may play a greater role in regulating diversity-biomass relationships than empirical studies have considered to date. In particular, the effect of competitive dominance on diversity-biomass relationships is not limited to covariance between relative fitness and monoculture biomass, as is suggested by reports on the "selection effect" of diversity. The existence of any RFD reduces the RYT in the simplest model we examined, even among consumers with identical monoculture yields that lack a highest performing species.

We attribute the positive effect on consumer biomass of reducing relative fitness differences to indirect effects propagating from dominant consumers. Any increase in one consumer's biomass cascades through the consumer-resource network, first decreasing the biomass of consumers of shared resources, and then increasing the biomass of those consumers' unshared resources. As one consumer with a fitness advantage becomes dominant, some fraction of the resources primarily consumed by subordinate species remains unused. Overall, less of the resource pool is converted into consumer biomass, reducing the total biomass in comparison to that obtained under a more equitable distribution of fitness, but identical resource partitioning.

Another departure from prior theory revealed by our analysis is that overexploitation of resources can cause large deviations from the pattern of positive diversitybiomass correlations. Without resource extinction, the dynamics of MacArthur's consumers could be put into Lotka-Volterra form by appealing to a separation of resource and consumer timescales. Thus, our special case corresponds closely to Loreau's (2004) analysis of the RYT in Lotka-Voleterra systems. However, allowing resource extinction moves Eq. 1 outside the scope of a Lotka-Voleterra system and Loreau's analysis. The communities with relatively low RYT values interspersed throughout Fig. 1B are precisely those in which resources go extinct in consumer monocultures. Close examination of these cases reveals the mechanism, which we describe for the simplest case of two consumers and two resources. A single consumer that overexploits its primary resource, driving it to extinction, can retain a high equilibrium biomass in a monoculture supported by the second resource. However, when competition forces this consumer to rely on its primary resource, the consumer can only persist at low density; otherwise the resource goes extinct as previously described. In this way, the consumer's relative yield drops dramatically in the presence of a competitor. Such scenarios offer important exceptions to more commonly reported positive effects of biodiversity on biomass yield, and again reveal that competitive dominance can drastically affect diversity-function relationships.

Our theoretical observations suggest that greater care must be taken in the analysis of biodiversity-function experiments. The multiple indirect interactions between consumers which compel attention to the effects of relative fitness differences make the goal of an additive partition of diversity effects appear elusive, and possibly unattainable. Indeed, we see no possibility for such a partition in the simple mechanistic model of competition considered here. Consequently, the post hoc statistical methods currently used to discern the mechanisms that drive effects of diversity on biomass do not necessarily reflect real biological processes that relate to mechanisms of species coexistence. Although we do not argue entirely against their use in attempting to discern how random vs. selective species loss may affect ecosystem function, these metrics cannot be equated to biological mechanisms.

Lastly, we note that our work relates community biomass to the niche and relative fitness differences in communities with fixed levels of diversity. Future theoretical work should tackle a more general relationship predicting community biomass from three independent variables: niche differences, relative fitness differences, and species richness. Empirical work can proceed in parallel. Coupling the experimental manipulations we have described with the standard design for measuring effects of diversity on ecosystem functions would empirically show how mechanisms of species coexistence control the functional consequences of biodiversity.

\section{ACKNOWLEDGMENTS}

Jonathan Levine, Jeremy Fox, and two anonymous reviewers provided superb critiques of the manuscript. Funding came from the U.S. National Science Foundation as a Graduate Research Fellowship to I. T. Carroll, grant EF-0742521 to R. M. Nisbet, and grants DEB-0614428/DEB-0842009 to B. J. Cardinale.

\section{Literature Cited}

Aarssen, L. W. 1997. High productivity in grassland ecosystems: effected by species diversity or productive species? Oikos 80:183-184.

Abrams, P. A. 1975. Limiting similarity and the form of the competition coefficient. Theoretical Population Biology 8:356-375.

Abrams, P. A., C. Rueffler, and R. Dinnage. 2008. Competition-similarity relationships and the nonlinearity of competitive effects in consumer-resource systems. American Naturalist 172:463-474.

Adler, P. B., J. HilleRisLambers, and J. M. Levine. 2007. A niche for neutrality. Ecology Letters 10:95-104.

Armstrong, R. A., and R. McGehee. 1980. Competitive exclusion. American Naturalist 115:151-170.

Balvanera, P., A. B. Pfisterer, N. Buchmann, J. He, T. Nakashizuka, D. Raffaelli, and B. Schmid. 2006. Quantifying the evidence for biodiversity effects on ecosystem functioning and services. Ecology Letters 9:1146-1156.

Cadotte, M. W., B. J. Cardinale, and T. H. Oakley. 2008. Evolutionary history and the effect of biodiversity on plant 
productivity. Proceedings of the National Academy of Sciences USA 105:17012-17017.

Cardinale, B. J., D. S. Srivastava, J. E. Duffy, J. P. Wright, A. L. Downing, M. Sankaran, and C. Jouseau. 2006. Effects of biodiversity on the functioning of trophic groups and ecosystems. Nature 443:989-992.

Cardinale, B. J., J. P. Wright, M. W. Cadotte, I. T. Carroll, A. Hector, D. S. Srivastava, M. Loreau, and J. J. Weis. 2007. Impacts of plant diversity on biomass production increase through time because of species complementarity. Proceedings of the National Academy of Sciences USA 104:1812318128.

Chapin, F. S., B. H. Walker, R. J. Hobbs, D. U. Hooper, J. H. Lawton, O. E. Sala, and D. Tilman. 1997. Biotic control over the functioning of ecosystems. Science 277:500-504.

Chesson, P. 1990. MacArthur's consumer-resource model. Theoretical Population Biology 37:26-38.

Chesson, P. 1994. Multispecies competition in variable environments. Theoretical Population Biology 45:227-276.

Chesson, P. 2000. Mechanisms of maintenance of species diversity. Annual Review of Ecology and Systematics 31:343-366.

Díaz, S., and M. Cabido. 2001. Vive la différence: plant functional diversity matters to ecosystem processes. Trends in Ecology and Evolution 16:646-655.

Edwards, K. F., and S. J. Schreiber. 2010. Preemption of space can lead to intransitive coexistence of competitors. Oikos 119:1202-1209.

Fox, J. W. 2005. Interpreting the "selection effect" of biodiversity on ecosystem function. Ecology Letters 8:846856.

Harpole, W. S., and K. N. Suding. 2007. Frequency-dependence stabilizes competitive interactions among four annual plants. Ecology Letters 10:1164-1169.

Haygood, R. 2002. Coexistence in MacArthur-style consumerresource models. Theoretical Population Biology 61:215-223.

Hector, A. 1998. The effect of diversity on productivity: detecting the role of species complementarity. Oikos 82:597-599.

Hector, A., T. Bell, J. Connolly, J. Finn, J. W. Fox, L. Kirwan, M. Loreau, J. McLaren, B. Schmid, and A. Weigelt. 2009. The analysis of biodiversity experiments: from pattern toward mechanism. Pages $94-104$ in S. Naeem, D. E. Bunker, A. Hector, M. Loreau, and C. Perrings, editors. Biodiversity, ecosystem functioning, and human wellbeing: an ecological and economic perspective. Oxford University Press, Oxford, UK.

Hooper, D. U., and P. M. Vitousek. 1997. The effects of plant composition and diversity on ecosystem processes. Science 277:1302-1305.

Huston, M. A. 1997. Hidden treatments in ecological experiments: re-evaluating the ecosystem function of biodiversity. Oecologia 110:449-460.

Jansen, V. A. A., and K. Sigmund. 1998. Shaken not stirred: on permanence in ecological communities. Theoretical Population Biology 54:195-201.
Leibold, M. A. 1995. The niche concept revisited: mechanistic models and community context. Ecology 76:1371-1382.

Levine, J. M., and J. HilleRisLambers. 2009. The importance of niches for the maintenance of species diversity. Nature 461:254-257.

Loreau, M. 2004. Does functional redundancy exist? Oikos 104:606-611.

Loreau, M., and A. Hector. 2001. Partitioning selection and complementarity in biodiversity experiments. Nature 412:7276.

Loreau, M., S. Naeem, P. Inchausti, J. Bengtsson, J. P. Grime, A. Hector, D. U. Hooper, M. A. Huston, D. Raffaelli, B. Schmid, D. Tilman, and D. A. Wardle. 2001. Biodiversity and ecosystem functioning: current knowledge and future challenges. Science 294:804-808.

MacArthur, R. 1970. Species packing and competitive equilibrium for many species. Theoretical Population Biology 1:111.

MacArthur, R. H., and R. Levins. 1967. The limiting similarity, convergence, and divergence of coexisting species. American Naturalist 101:377-385.

May, R. M. 1974. On the theory of niche overlap. Theoretical Population Biology 5:297-332.

Meszena, G., M. Gyllenberg, L. Pasztor, and J. A. J. Metz. 2006. Competitive exclusion and limiting similarity: a unified theory. Theoretical Population Biology 69:68-87.

Mouquet, N., J. L. Moore, and M. Loreau. 2002. Plant species richness and community productivity: why the mechanism that promotes coexistence matters. Ecology Letters 5:56-65.

Petchey, O. L. 2003. Integrating methods that investigate how complementarity influences ecosystem functioning. Oikos 101:323-330.

Sala, O. E. 2000. Global biodiversity scenarios for the year 2100. Science 287:1770-1774.

Schoener, T. W. 1976. Alternatives to Lotka-Volterra competition: models of intermediate complexity. Theoretical Population Biology 10:309-333.

Stachowicz, J. J., J. F. Bruno, and J. E. Duffy. 2007. Understanding the effects of marine biodiversity on communities and ecosystems. Annual Review of Ecology, Evolution, and Systematics 38:739-766.

Thompson, R., and B. Starzomski. 2007. What does biodiversity actually do? A review for managers and policy makers. Biodiversity and Conservation 16:1359-1378.

Tilman, D. 1999. The ecological consequences of changes in biodiversity: a search for general principles. Ecology 80:1455-1474.

Tilman, D., C. L. Lehman, and K. T. Thomson. 1997. Plant diversity and ecosystem productivity: theoretical considerations. Proceedings of the National Academy of Sciences USA 94:1857-1861.

Vandermeer, J. H. 1989. The ecology of intercropping. Cambridge University Press, Cambridge, UK.

Warren, P. H., R. Law, and A. J. Weatherby. 2003. Mapping the assembly of protest communities in microcosms. Ecology 84:1001-1011.

\section{APPENDIX A}

Potential ambiguity in the calculation of niche and relative fitness differences and limitations on their interpretation (Ecological Archives E092-094-A1).

\section{APPENDIX B}

Relating the equilibrium of MacArthur's model to the niche difference (ND), relative fitness difference (RFD), and relative yield total (RYT) (Ecological Archives E092-094-A2).

\section{APPENDIX C}

Methods and additional results for the numerical analysis (Ecological Archives E092-094-A3). 


\section{ERRATA}

Richard B. Chandler has reported three errors in the paper by Chandler et al., published in the July 2011 issue (Chandler, R. B., J. A. Royle, and D. I. King. 2011. Inference about density and temporary emigration in unmarked populations. Ecology 92:1429-1435). First, the expression $J \equiv 1$ following Eq. 1 should be $T \equiv 1$.

Additionally, Eq 2 should be changed from

$$
\begin{aligned}
& L\left(\lambda, \phi, p \mid \mathbf{y}_{i t}\right)= \\
& \prod_{i}^{R}\left\{\sum_{M_{i}=\max \left(\mathbf{y}_{i t .}\right)}^{\infty}\left[\sum_{N_{i t}=y_{i t .}}^{M_{i}}\left(\frac{N_{i t} !}{y_{i t 1} ! y_{i t 2} ! y_{i t 3} ! y_{i t 0} !} \pi_{1}^{y_{i t 1}} \pi_{2}^{y_{i t 2}} \pi_{3}^{y_{i t 3}} \pi_{0}^{N_{i t}-y_{i t}}\right) g\left(N_{i t} \mid M_{i}, \phi\right)\right] f\left(M_{i} \mid \lambda\right)\right\}
\end{aligned}
$$

to

$$
\begin{aligned}
& L(\lambda, \phi, p \mid \mathbf{y})= \\
& \prod_{i=1}^{R}\left\{\sum_{M_{i}=\max \left(\mathbf{y}_{i t .}\right)}^{\infty}\left(\prod_{t=1}^{T}\left[\sum_{N_{i t}=y_{i t .}}^{M_{i}} \frac{N_{i t} !}{y_{i t 1} ! y_{i t 2} ! y_{i t 3} ! y_{i t 0} !} \pi_{1}^{y_{i t 1}} \pi_{2}^{y_{i t 2}} \pi_{3}^{y_{i t 3}} \pi_{0}^{N_{i t}-y_{i t .}} g\left(N_{i t} \mid M_{i}, \phi\right)\right]\right) f\left(M_{i} \mid \lambda\right)\right\}
\end{aligned}
$$

Finally, Eq. 3 should be changed from

$$
\begin{aligned}
& L\left(\lambda, \phi, p \mid \mathbf{y}_{i t}\right)= \\
& \qquad \prod_{i=1}^{R}\left\{\sum_{M_{i}=\max \left(\mathbf{y}_{i t .}\right)}^{\infty}\left(\frac{M_{i} !}{y_{i 1} ! y_{i 2} ! y_{i 3} ! y_{i 0} !}\left(\phi \pi_{1}\right)^{y_{i 1}}\left(\phi \pi_{2}\right)^{y_{i 2}}\left(\phi \pi_{3}\right)^{y_{i 3}}\left(\phi \pi_{0}\right)^{M_{i}-\mathbf{y}_{i t}}\right) f\left(M_{i} \mid \lambda\right)\right\}
\end{aligned}
$$

to

$$
\begin{aligned}
& L(\lambda, \phi, p \mid \mathbf{y})= \\
& \prod_{i=1}^{R}\left\{\sum_{M_{i}=\max \left(\mathbf{y}_{i t .}\right)}^{\infty}\left(\prod_{t=1}^{T} \frac{M_{i} !}{y_{i t 1} ! y_{i t 2} ! y_{i t 3} ! y_{i t 0} !}\left(\phi \pi_{1}\right)^{y_{i t 1}}\left(\phi \pi_{2}\right)^{y_{i t 2}}\left(\phi \pi_{3}\right)^{y_{i t 3}}\left(\phi \pi_{0}\right)^{M_{i}-y_{i t .}}\right) f\left(M_{i} \mid \lambda\right)\right\}
\end{aligned}
$$

The authors thank Robert Dorazio for calling these mistakes to their attention.

Ian Carroll has reported an error in Eq. 3 of the article by Carroll et al. in the May 2011 issue (Carroll, I. T., B. J. Cardinale, and R. M. Nisbet. 2011. Niche and fitness differences relate the maintenance of diversity to ecosystem function. Ecology 92:1157-1165). The second overbar in the expression on the right-hand side of the equation should not extend over the " 2 " exponent. The corrected expression is as follows:

$$
\mathrm{RFD}=\exp \left[\left(\overline{(\ln S)^{2}}-\overline{\ln S}^{2}\right)^{1 / 2}\right]
$$

